\title{
Quantification of the orientations of pyrrolidine-based oxypeptide nucleic acid-DNA hybrid duplexes
}

\author{
Mizuki Kitamatsu* and Masahiko Sisido \\ Received (in $X X X, X X X) X$ th $X X X X X X X X X 200 X$, Accepted Xth $X X X X X X X X X 200 X$ \\ ${ }_{5}$ First published on the web Xth $X X X X X X X X X 200 X$ \\ DOI: $10.1039 / b 000000 x$
}

We describe the fluorescence quenching-based quantification of complementary parallel and antiparallel hybrids of DNAs with pyrrolidine-based oxypeptide nucleic acids (POPNAs). When BODIPY-modified DNAs as fluorescent probe formed hybrids with complementary POPNAs, 10 fluorescence of the BODIPY was effectively quenched by the guanine unit and the Lys unit on the POPNAs. The orientations of hybrids of POPNA with DNA were estimated by the quenching efficiencies of two BODIPY-modified DNAs. As the results, we clarified that configurations of POPNAs affect the extent of orientation of the hybrid duplexes.

Oxypeptide nucleic acids (OPNAs) ${ }^{1}$ and the related 15 conformationally restricted pyrrolidine-based OPNAs (POPNAs) $^{2}$ are DNA surrogates that bind to complementary oligonucleotides with moderate affinity and sequence specificity (Fig. 1). When hybridized to complementary oligonucleotides, the OPNAs and POPNAs have very sharp 20 melting curves. The OPNAs and POPNAs also have high water solubility relative to Nielsen-type peptide nucleic acids (PNAs, Fig. 1$)^{3}$, and this is due to the ether linkages in the main chains. These characteristics of OPNAs and POPNAs are important properties that enable identification of single

25 mismatches in oligonucleotide sequences in aqueous media and as such, provide useful biomedical tools and medicine based on the peptide nucleic acids.

In addition to these properties, the orientation of hybrids between peptide nucleic acids and oligonucleotides (i.e., 30 whether the $\mathrm{N}$-terminus of a hybridized peptide nucleic acid is facing the 5'-end [parallel orientation] or the 3'-end [antiparallel orientation] of an oligonucleotide) is also an important factor to be considered in discussions of hybrid structures and in the design of optimal base sequences for 35 hybridization to various oligonucleotide sequences. In the nucleic acid medicine without side effect, the orientation is especially important to design the base sequences. The preferred orientations of peptide nucleic acid/oligonucleotide pairs are generally evaluated by comparison of melting 40 temperatures $\left(T_{\mathrm{m}} \mathrm{s}\right)$ of the duplex formed with complementary oligonucleotides in the parallel orientation and the antiparallel orientation ${ }^{4}$. However, the preferred orientation of the hybrids estimated by this method is qualitative and does not enable quantification of the individual content of the two orientations 45 of a given hybrid. Furthermore, this method cannot be used to evaluate the preferred orientation of hybrids involving biologically important palindromic DNA sequences.

In this study, we quantified the fraction of orientation of the hybrids of POPNAs with DNAs by using quenching of
50 fluorescence tag-modified DNAs induced by the presence of a guanine moiety linked to the POPNAs. The POPNAs contain pyrrolidine rings in the main chain and the pyrrolidine ring has 2 chiral centers, which provide a total of 4 configurations (cis-L-, trans-L-, cis-D-, and trans-D-). The POPNAs form 55 hybrids with DNA and RNA depending on the configurations of the pyrrolidine rings. The different configurations of POPNAs are expected to influence the content of the 2 orientations of the DNA hybrids.

\section{"Figure 1 here"}

60 Fig. 1 Chemical structures of OPNA, POPNA, DNA, and Nielsen-type PNA.

To quantify the fraction of orientation of hybrids of POPNAs with complementary DNAs, we selected a BODIPY as the fluorophore (hereafter Bdp; 4,4-difluoro-5,7-dimethyl65 4-bora-3a,4a-diaza-5-indacene-3-propionyl group). First, Bdp moieties linked at the 5'- or 3'-end of 9-mer thymine DNAs with an intervening cytosine unit (Bdp-dCT9 and dT9C-Bdp, respectively) were purchased from Greiner BioOne (Germany). We prepared a 9-mer of adenine trans-D-POPNA with an N70 terminal Lys unit and a C-terminal acetylated guanine POPNA residue (G- $t \mathrm{D}-\mathrm{A} 9-\mathrm{K}$, Fig. 2). The latter is provided as a quencher of $\mathbf{B d p}^{5}$. The sequence is Ac-G-AAAAAAAAALys- $\mathrm{NH}_{2}$, where $G, \mathrm{~A}$, and Lys indicate a guanine unit of OPNA, an adenine unit of trans-D-POPNA, and lysine, 75 respectively. The $\mathrm{N}$-terminal Lys is a primary amine and the C-terminal guanine is acetylated. We also prepared a 9-mer adenine trans-D-POPNA modified with a Lys unit at the Cterminus (tD-A9-K, sequence: Ac-AAAAAAAAA-Lys- $\mathrm{NH}_{2}$, Fig. 2) as a control (without the guanine quencher). For ${ }_{80}$ example, if G-tD-A9-K effectively quenches fluorescence of dT9C-Bdp, but not of Bdp-dCT9, an antiparallel orientation is expected. Fluorescence spectra of 
Table 1: Quantification of the fraction of parallel $\left(f_{p}\right)$ and antiparallel $\left(f_{a p}\right)$ orientations from quenching efficiencies of Bdp-dCT9 and dT9C-Bdp by various combinations of 9-mer adenine pyrrolidine-based oxypeptide nucleic acid (POPNA) configurations with 9-mer thymine DNA.

\begin{tabular}{|c|c|c|c|c|c|c|}
\hline \multirow{2}{*}{ POPNA and DNA as quenchers } & \multicolumn{2}{|c|}{ Quenching efficiency for } & \multirow{2}{*}{$T_{\mathrm{m}}\left({ }^{\circ} \mathrm{C}\right)^{a}$} & \multirow{2}{*}{$\mathrm{f}_{\mathrm{p}}$} & \multirow{2}{*}{$\mathrm{f}_{\text {ap }}$} & \multirow{2}{*}{$\mathrm{f}_{\mathrm{ap}} /\left(\mathrm{f}_{\mathrm{p}}+\mathrm{f}_{\mathrm{ap}}\right)$} \\
\hline & Bdp-dCT9 & dT9C-Bdp & & & & \\
\hline G-tD-A9-K & 0.52 & 0.68 & 26 & $0.38^{b}$ & $0.62^{b}$ & 0.62 \\
\hline$t \mathrm{D}-\mathrm{A} 9-\mathrm{K}$ & 0.21 & 0.08 & 23 & $0.29^{c}$ & $0.71^{c}$ & 0.71 \\
\hline$c$ D-A9-K & 0.20 & 0.08 & 22 & $0.29^{c}$ & $0.68^{c}$ & 0.70 \\
\hline$t \mathrm{~L}-\mathrm{A} 9-\mathrm{K}$ & 0.06 & 0.01 & 19 & $0.01^{c}$ & $0.20^{c}$ & 0.95 \\
\hline cL-A9-K & 0.26 & 0.03 & 26 & $0.12^{c}$ & $0.87^{c}$ & 0.88 \\
\hline d(A9G) & 0.88 & 0.03 & 14 & $0.03^{d}$ & $0.97^{d}$ & 0.97 \\
\hline d(GA9) & 0.07 & 0.84 & 12 & $0.08^{d}$ & $0.92^{d}$ & 0.92 \\
\hline
\end{tabular}

${ }^{a}$ These values were estimated from melting curves recorded upon heating the solution at $0.5^{\circ} \mathrm{C} / 0.5 \mathrm{~min}$. Essentially the same curves were obtained in the cooling process. Conditions: fluorescence was monitored at $514 \mathrm{~nm}$ with duplexes in aqueous buffer with $100 \mathrm{mM} \mathrm{NaCl}^{\circ} 10 \mathrm{mM} \mathrm{NaH}_{2} \mathrm{PO}_{4}$, and $0.1 \mathrm{mM}$ ${ }_{5}$ EDTA, $\left.\mathrm{pH} 7.0\right)$. [POPNA $]=[\mathrm{DNA}]=1 \mu \mathrm{M} .{ }^{b}$ Calculated from equations $1-6$. The $\mathrm{q}_{\mathrm{G}}$ and $\mathrm{q}_{\mathrm{K}}$ values were 0.91 and 0.29 , respectively. ${ }^{c}$ Calculated using $\mathrm{q}_{\mathrm{K}}$ $=0.29$, without constraints on the $\left(\mathrm{f}_{\mathrm{p}}+\mathrm{f}_{\mathrm{ap}}\right)$ value. ${ }^{d}$ Calculated using $\mathrm{q}_{\mathrm{G}}=0.91$, without constraints on the $\left(\mathrm{f}_{\mathrm{p}}+\mathrm{f}_{\mathrm{ap}}\right)$ value.

G-tD-A9-K/dT9C-Bdp and G-tD-A9-K/Bdp-dCT9 before and after hybridization are shown in Fig. 3A. In both the cases, the fluorescence arising from Bdp $\left(50{ }^{\circ} \mathrm{C}\right)$ is decreased when the 10 hybrids are formed $\left(5^{\circ} \mathrm{C}\right)^{\mathrm{i}}$. However, fluorescence of the G-tDA9-K/dT9C-Bdp pair appears to be more effectively quenched than that of the G-tD-A9-K/Bdp-dCT9 pair. This indicates a preference of antiparallel orientation for the G-tDA9-K/dT9 hybrid duplex. Furthermore, the temperature 15 dependence of the fluorescence intensity at $514 \mathrm{~nm}$ emitted from Bdp of these mixtures was measured (Fig. 3B).

The melting curve of G-tD-A9-K/Bdp-dCT9 mixture is in accord with that of the G-tD-A9-K/dT9C-Bdp mixture. Each hybrid resulted in well-defined sigmoidal melting curves by 20 that be similar to 9-mer adenine trans-D-POPNA/dT9 mixture (see Fig. S1). These results clearly indicate that the quenching behavior is due to formation of the hybrid duplexes ${ }^{\mathrm{ii}}$. These results are also supported by the $1 / 1$ duplex formation obtained in the titration experiments of the mixtures (see Fig. ${ }_{25}$ 2S).

\section{"Figure 2 here"}

Fig. 2 Chemical structures of G-tD-A9-K and tD-A9-K.

However, the quantitative evaluation of the content of the 2 orientations was complicated by the observation that $t \mathrm{D}-\mathrm{A} 9-\mathrm{K}$ 30 also quenches the fluorescence of Bdp-dCT9 and dT9C-Bdp to some extent with the quenching caused by the C-terminal Lys unit of the $t \mathrm{D}-\mathrm{A} 9-\mathrm{K}$. Indeed, $t \mathrm{D}-\mathrm{A} 9-\mathrm{K}$ quenches fluorescence of Bdp-dCT9 more effectively than dT9C-Bdp, due to the close proximity of the Lys unit to the Bdp of the ${ }_{35}$ Bdp-dCT9 in an antiparallel hybrid (Fig. 3C). The temperature dependence of the fluorescence intensity from Bdp of these mixtures was also in fair agreement with the melting curves of the G-tD-A9-K/dT9s mixtures shown in Fig. 3B (Fig. 3D). Consequently, we must evaluate the extent of 40 orientation of the hybrids by considering the effect of quenching of Bdp by the Lys unit.

\section{"Figure 3 here"}

Fig. 3 (A) Fluorescence spectra of the mixtures of G-tD-A9-K with the complementary Bdp-dCT9 and dT9C-Bdp, (B) Temperature dependence 45 for fluorescence intensity of the mixtures of G-tD-A9-K with the complementary Bdp-dCT9 (open circles) and dT9C-Bdp (closed circles), (C) Fluorescence spectra of the mixtures of $t \mathrm{D}-\mathrm{A} 9-\mathrm{K}$ with the complementary Bdp-dCT9 and dT9C-Bdp, and (D) Temperature dependence for the fluorescence intensity of the mixtures of $t \mathrm{D}-\mathrm{A} 9-\mathrm{K}$ with 50 the complementary Bdp-dCT9 (open circles) and dT9C-Bdp (closed circles). Conditions: $\lambda_{\mathrm{ex}}=495 \mathrm{~nm}$. In aqueous buffer (100 mM NaCl, 10 $\mathrm{mM} \mathrm{NaH}{ }_{2} \mathrm{PO}_{4}$, and $0.1 \mathrm{mM}$ EDTA, pH 7.0). [G-tD-A9-K] = [Bdp-dCT9] $=[\mathrm{dT} 9 \mathrm{C}-\mathbf{B d p}]=1 \mu \mathrm{M}$. These spectra and melting curves were recorded upon cooling of the solution. Essentially the same curves were obtained 55 during the heating process.

To evaluate the fractions of parallel and antiparallel orientation of G-tD-A9-K/dT9 pairs ( $f_{p}$ ' and $f_{a p}$ ', respectively), and those of $t \mathrm{D}-\mathrm{A} 9-\mathrm{K} / \mathrm{dT}$ 9pairs ( $\mathrm{f}_{\mathrm{p}}$ and $\mathrm{f}_{\mathrm{ap}}$, respectively), the following relationships were derived. For quenching of Bdp${ }_{60} \mathrm{dCT} 9$ and dT9C-Bdp by hybridization with G-tD-A9-K, the quenching efficiency of the G-tD-A9-K/Bdp-dCT9 pair $\left(\mathrm{Q}_{1}\right)$ and the G-tD-A9-K/dT9C-Bdp pair $\left(Q_{2}\right)$ are expressed as follows:

65

$$
\begin{aligned}
& \mathrm{Q}_{1}=\mathrm{q}_{\mathrm{G}} \mathrm{f}_{\mathrm{p}}{ }+\mathrm{q}_{\mathrm{K}} \mathrm{f}_{\text {ap }} \\
& \mathrm{Q}_{2}=\mathrm{q}_{\mathrm{K}} \mathrm{f}_{\mathrm{p}}{ }_{\mathrm{p}}+\mathrm{q}_{\mathrm{G}} \mathrm{f}^{\prime} \text { ap }
\end{aligned}
$$

Also, for quenching of Bdp-dCT9 and dT9C-Bdp by hybridization with $t \mathrm{D}-\mathrm{A} 9-\mathrm{K}$, the quenching efficiency of the tD-A9-K/Bdp-dCT9 pair $\left(\mathrm{Q}_{3}\right)$ and the $t \mathrm{D}-\mathrm{A} 9-\mathrm{K} / \mathrm{dT} 9 \mathrm{C}-\mathrm{Bdp}$ pair $\left(\mathrm{Q}_{4}\right)$ are expressed as follows:

70

$$
\begin{aligned}
& \mathrm{Q}_{3}=\mathrm{q}_{\mathrm{K}} \mathrm{f}_{\mathrm{ap}} \\
& \mathrm{Q}_{4}=\mathrm{q}_{\mathrm{K}} \mathrm{f}_{\mathrm{p}}
\end{aligned}
$$

In the above equations, $\mathrm{q}_{\mathrm{G}}$ denotes the quenching efficiency for a pair of Bdp units and a guanine unit located on the same side of a hybrid. Similarly, $\mathrm{q}_{\mathrm{K}}$ denotes the quenching 75 efficiency for a pair of Bdp units and Lys unit. Since $T_{\mathrm{m}} \mathrm{S}$ of tD-A9-K/dT9 and G-tD-A9-K/dT9 pairs are well above the temperature required for fluorescence measurements $\left(5^{\circ} \mathrm{C}\right)$, the sum of the parallel and antiparallel fractions must be unity.

$$
\begin{gathered}
\mathrm{f}_{\text {ap }}{ }^{\prime}+\mathrm{f}_{\mathrm{p}}{ }^{\prime}=1 \\
\mathrm{f}_{\text {ap }}+\mathrm{f}_{\mathrm{p}}=1
\end{gathered}
$$

By inserting the experimental quenching efficiencies into the above equations, the 6 variables $\left(\mathrm{f}_{\mathrm{p}}, \mathrm{f}_{\mathrm{ap}}, \mathrm{f}_{\mathrm{p}}, \mathrm{f}_{\mathrm{ap}}, \mathrm{q}_{\mathrm{G}}\right.$, and $\mathrm{q}_{\mathrm{K}}$ ) were determined as listed in Table 1 . As expected from the qualitative discussion above, the antiparallel orientation ${ }_{85}(62 \%)$ is preferred for the G-tD-A9-K/dT9 pair, where, $\mathrm{q}_{\mathrm{G}}=$ 0.91 , whereas $\mathrm{q}_{\mathrm{K}}=0.29$. ${ }^{\mathrm{iii}}$ The value quantified from the $t \mathrm{D}$ - 
A9-K/dT9 pair also showed that the antiparallel orientation (71\%) is preferred.

Since Bdp is quenched by the Lys, for the remaining configurations of POPNA, the preferred orientation was 5 quantified from the quenching of Bdp by the Lys unit at the C-terminus of the control POPNAs (without the guanine unit) by using $\mathrm{q}_{\mathrm{K}}=0.29$ as determined for their hybrids. We prepared cis-D-, trans-L-, and cis-L-configured 9-mer adenine POPNA with the C-terminal Lys (cD-A9-K, $t \mathrm{~L}-\mathrm{A} 9-\mathrm{K}$, and $c \mathrm{~L}-$ $10 \mathrm{~A} 9-\mathrm{K}$, respectively) and quantified the fractions of orientation of these hybrid duplexes with dT9 by the aforementioned protocols. The results are shown in Table 1. As references, the dA9/dT9 (hybrid duplexes of d(GA9) or d(A9G) with BdpdCT9 or dT9C-Bdp) were also used to quantify the fractions 15 of the 2 orientations (see Fig. S3). These results are also listed in Table 1. All POPNAs favored the antiparallel orientation with the DNA but the parallel orientation is also allowed to some extent. In contrast, nearly complete antiparallel orientation of DNA/DNA pairs is observed (the fraction of 20 antiparallel orientation of the dA9/dT9 hybrid is 92-97\%). The L-configured POPNAs (cis-L: 88\%, trans-L: $95 \%{ }^{\mathrm{iv}}$ ) preferred antiparallel orientation to a greater extent than the Dconfigured POPNAs (cis-D: 70\%, trans-D: 71\%). These results indicate that configurations of POPNAs do not only affect 25 hybrid stability of POPNAs with oligonucleotides, but also affect the extent of orientation of the hybrid duplexes.

\section{Conclusions}

We successfully quantified the fractional orientations of hybrid duplexes of POPNAs with DNAs by using 30 fluorescence quenching of the Bdp fluorophore, which occurs upon formation of the POPNA-DNA hybrid duplexes. The preferred orientation of these hybrids is the same antiparallel orientation as the DNA/DNA hybrid and the PNA/DNA hybrid. However, among POPNAs with different 35 configurations, the content of the 2 different orientations was different. L-Configured POPNA/DNA hybrids prefer the parallel orientation over the antiparallel orientation to a greater extent than the D-configured POPNA/DNA hybrid duplexes. The method for quantification of the orientation of 40 hybrids can be also used for other artificial nucleic acids. This method will be useful to design more effective nucleic acid medicine.

\section{Notes and references}

Department of Medical and Bioengineering, Graduate School of Natural 45 Science and Technology, Okayama University, 3-1-1 Tsushimanaka, kitaku, Okayama 700-0082, Japan. Tel/Fax: +81-86-251-8219; E-mail:

kitamatu@cc.okayama-u.ac.jp

† Electronic Supplementary Information (ESI) available: [Melting curves of POPNA/DNA pairs, fluorescence titration curves of G-tD-A9-K/Bdp50 dCT9 and G-tD-A9-K/dT9C-Bdp, and fluorescence spectra of DNA/DNA pairs]. See DOI: 10.1039/b000000x/

$\ddagger \quad$ i. The $T_{\mathrm{m}}$ of the hybrid of G-tD-A9-K and dT9 was $25^{\circ} \mathrm{C}$. See Table 1 .

ii. Since Bdp of DNA adjoins a guanine unit of POPNA in order to 55 form hybrid between DNA and POPNA, the Bdp is quenched by the guanine unit. iii. It is assumed that $\mathrm{q}_{\mathrm{G}}$ and $\mathrm{q}_{\mathrm{K}}$ are independent of the hybrid structures. These values indicate that Bdp is quenched by G and Lys enough in the hybrid.

60 iv. In this case, however, $\left(f_{p}+f_{a p}\right)$ was not set to unity, because the $T_{\mathrm{m}}\left(19{ }^{\circ} \mathrm{C}\right)$ of the trans-L-POPNAs/DNA pair are not always much higher than the temperature used for the fluorescence measurements $\left(5^{\circ} \mathrm{C}\right)$.

1 M. Kuwahara, M. Arimitsu and M. Sisido, J. Am. Chem. Soc., 1999, 65 121, 256; M. Kuwahara, M. Arimitsu, M. Shigeyasu, N. Saeki and M. Sisido, J. Am. Chem. Soc., 2001, 123, 4653.

2 M. Kitamatsu, M. Shigeyasu, T. Okada and M. Sisido, Chem. Commun., 2004, 1208; M. Kitamatsu, M. Shigeyasu and M. Sisido, Chem. Lett., 2005, 34, 1216; M. Kitamatsu, M. Shigeyasu, M. Saitoh and M. Sisido, Biopolymers (Peptide Science), 2006, 84, 267; M. Kitamatsu, T. Kashiwagi, R. Matsuzaki and M. Sisido, Chem. Lett., 2006, 35, 300; M. Kitamatsu, A. Takahashi, T. Ohtsuki and M. Sisido, Tetrahedron, 2010, 66, 9659; M. Kitamatsu, S. Kurami, T. Ohtsuki and M. Sisido, Bioorg. Med. Chem Lett., 2010, 21, 225; K. -H. 75 Altmann, D. Hüsken, B. Cuenoud and C. Garcia-Echeverria, Bioorg. Med. Chem. Lett., 2000, 10, 929.

3 P. E. Nielsen, Peptide nucleic acids -protocols and applications-, Horizon Bioscience, Norfolk, England, 2004.

4 K. Siriwong, P. Chuichay, S. Saen-oon, C. Suparpprom, T. Vilaivan 80 and S. Hannongbua, Biochem. Biophys. Res. Commun., 2008, 372, 765; T. Vilaivan and C. Srisuwannaket, Org. Lett., 2006, 8, 1897; M. Hollenstein and C. J. Leumann, J. Org. Chem., 2005, 70, 3205; V. A. Kumar and K. N. Ganesh, Acc. Chem. Res., 2005, 38, 404; T. Wada, N. Minamimoto, Y. Inaki and Y. Inoue, J. Am. Chem. Soc., 2000, 122, 85 6900; B. Hyrup, M. Egholm, P. E. Nielsen, P. Wittung, B. Nordén and O. Buchardt, J. Am. Chem. Soc., 1994, 116, 7964.

5 S.Kurata, T. Kanagawa, K. Yamada, M. Torimura, T. Yokomaku, Y. Kamagata, R. Kurane, Nucleic Acids Res., 2001, 29, e34. 

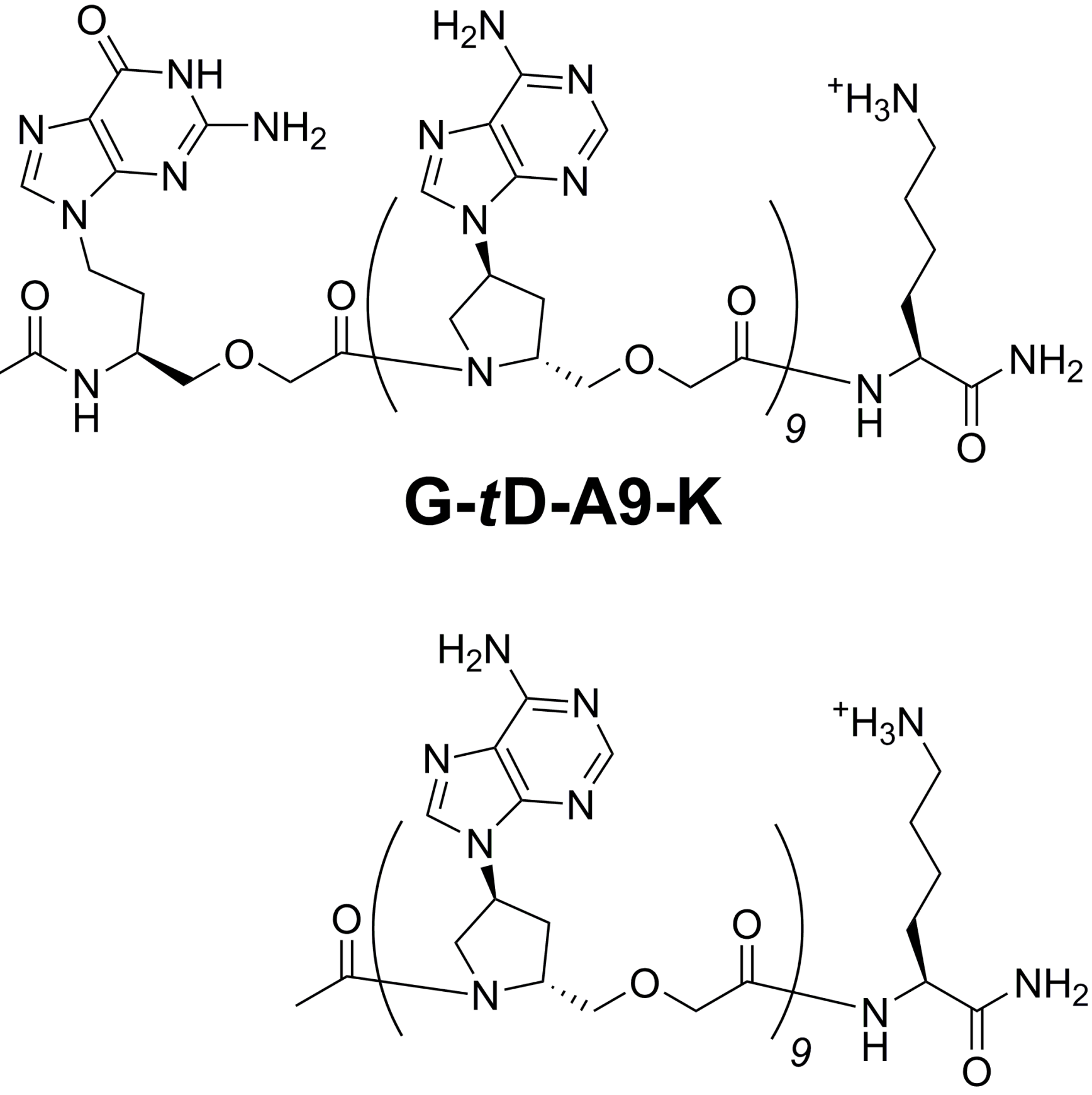

tD-A9-K 

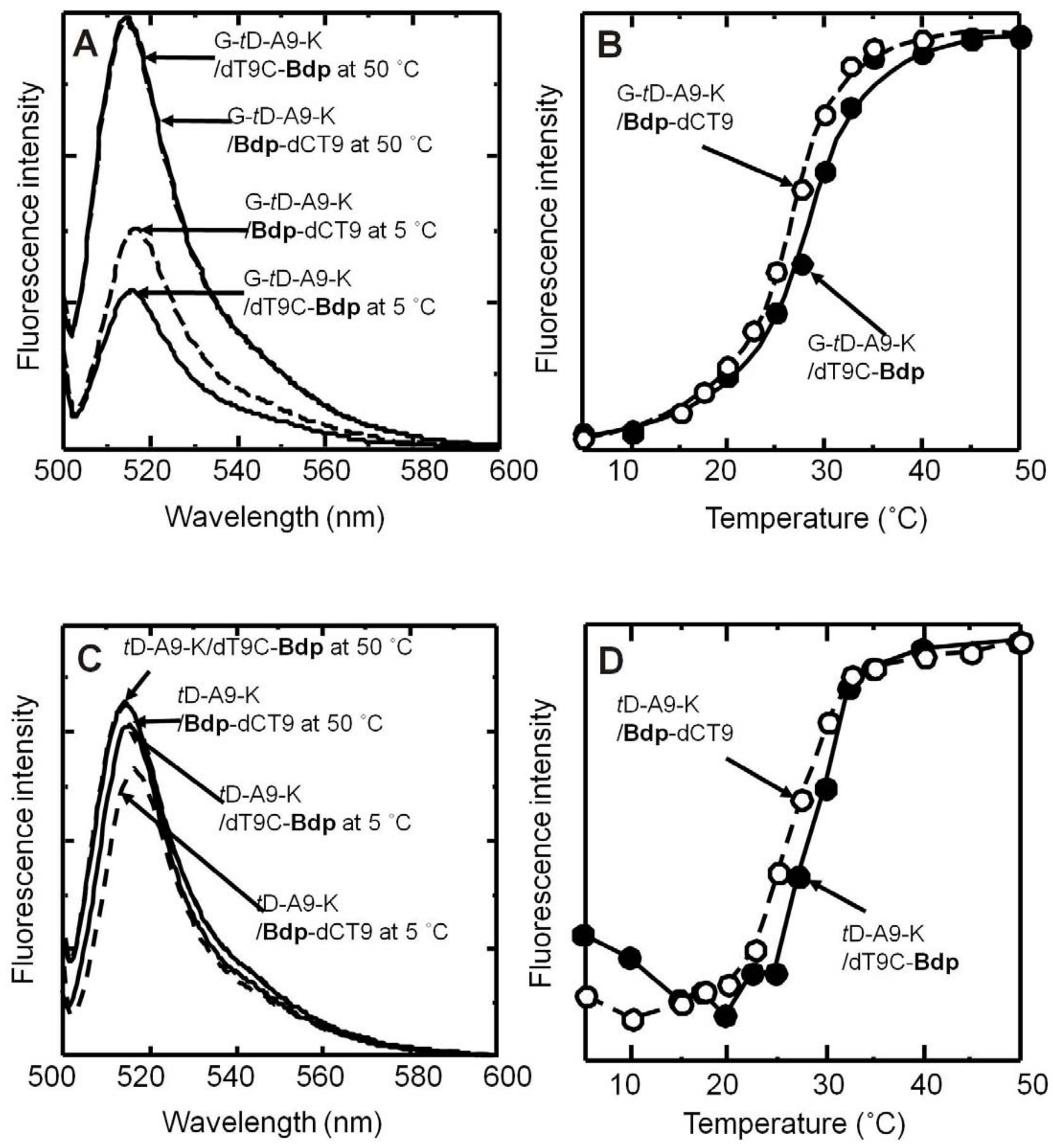